\title{
Novas regras e velhos desafios na regulação da propaganda de medicamentos
}

\section{| ${ }^{1}$ Regina Célia Borges Lucena |}

Resumo: Este estudo teve como objetivo analisar o processo de regulamentação da propaganda de medicamentos pela Agência Nacional de Vigilância Sanitária (Anvisa) ocorrido em 2008 e identificar os seus resultados quanto ao modelo regulador nessa área de atuação. Foram analisadas as mudanças ocorridas em 2008, com a publicação da RDC no 96/2008, a partir de três fontes de dados. A primeira foi a análise comparativa com a normativa anterior, a RDC no 102/2000.

Em seguida, as mudanças foram relacionadas a dois documentos técnicos, de escopos diferentes: os Critérios Éticos para a Promoção de Medicamentos da Organização Mundial de Saúde, de alcance mundial; e a Contribuição ao Debate e Proposiçôes à Consulta Pública 84/2005, de âmbito nacional, da Anvisa, relativa à propaganda de medicamentos no Brasil. A análise de onze mudanças de conteúdo na ordenação legal estabelecida pela RDC no 96/2008 aponta para uma maior proximidade com os Critérios Éticos para a Promoção de Medicamentos da Organização Mundial de Saúde, mas um claro distanciamento de proposiçôes para a alteração do modelo regulador atualmente estabelecido. Concluiu-se que a adoção de medidas que alterem o modelo regulador nesse campo de atuação, o da regulação da propaganda de medicamentos, requer mudanças mais substanciais no modelo de gestão e da ação regulatória da Anvisa, especialmente quanto ao seu papel de regulação social.

> Palavras-chave: propaganda de medicamentos; vigilância sanitária; regulação.
1 Mestre em Saúde Pública pela Fundação Oswaldo Cruz (2000): doutoranda em Política Social da Universidade de Brasília. Especialista em Regulação e Vigilância Sanitária da Agência Nacional de Vigilância Sanitária. Endereço eletrônico: reginalucena@unb.br
Recebido em: 08/12/2010. Aprovado em: 17/04/2012. 


\section{Introdução}

A complexificação do cuidado à saúde no período posterior à $2^{\text {a }}$ Guerra Mundial representou uma importante pressão sobre o aumento de custos dos sistemas de saúde em escala mundial. Desde então, diferentes fatores têm impactado ainda mais sobre esses custos, como a emergência de novas tecnologias, o envelhecimento populacional e aumento das expectativas da população sobre os serviços de saúde (DANIELS; SABIN, 2002). Ao lado disso, a mercantilização do cuidado à saúde levou à redefinição do papel dos indivíduos, quanto aos bens e serviços de saúde, de usuários para consumidores. Considera-se que essa mudança tem um impacto tão ou mais expressivo para o crescimento dos custos em saúde quanto os determinantes usualmente reconhecidos, como o envelhecimento (SYRETT, 2007).

É nesse contexto que os medicamentos assumiram, na lógica capitalista de produção, o papel de mercadoria, que, para além do uso terapêutico, cumpre a função de satisfazer expectativas de médicos e usuários (BARROS, 1983). A medicalização é definida como "a expansão progressiva do campo de intervenção da biomedicina por meio da redefinição de experiências e comportamentos humanos como se fossem problemas médicos" (TESSER, 2006, p. 62). O conceito de medicalização se popularizou na década de 70 , em referência à apropriação, pela medicina, de todas as etapas da vida humana, mesmo aquelas em que não há morbidade, como o parto e nascimento (CUNHA, 2008; SEIBERT et al., 2002).

O fenômeno da medicalização, enquanto intervenção na vida humana, estende-se aos medicamentos, potencializando o seu uso indiscriminado. Segundo a Organização Mundial de Saúde, o uso racional - e relacionado à necessidade - de medicamentos envolve requisitos como orientação e responsabilidade, qualidade dos medicamentos e condições para a sua adequação às necessidades dos usuários, tais como condições clínicas, dose e período apropriados, além de custo acessível (CUNHA, 2008). Portanto, está vinculado a questôes de segurança e à ocorrência de efeitos adversos, que correspondem a respostas prejudiciais a doses normalmente utilizadas, além de outros agravos à saúde relacionados ao uso irracional de medicamentos, como as intoxicações.

O perfil epidemiológico dos efeitos adversos a medicamentos ainda é pouco estudado no debate brasileiro em saúde pública (PFAFFENBACH et al., 2002). 
Ainda assim, diversos estudos, em diferentes períodos, confirmam o impacto dos

medicamentos na saúde humana, relacionando-o com seu uso indiscriminado (AQUINO, 2008; MENDONÇA; MARINHO, 2005; NASCIMENTO, 2003; ROZENFELD, 2007). A magnitude do problema das intoxicaçôes é sinalizada, ainda, pelo número de casos de intoxicações humanas por medicamentos, segundo registros no Sistema Nacional de Informações Toxico-Farmacológicas (Sinitox), da Fundação Oswaldo Cruz/Ministério da Saúde. Os dados do sistema em 2007 mostram que os medicamentos continuam representando parcela significativa das intoxicações humanas, com 21.375 casos registrados naquele ano, que correspondem a mais de $30 \%$ do total de registros.

Além dos agravos à saúde, a crescente demanda judicial por medicamentos após a Constituição Federal de 1988 revela outro impacto decorrente do aumento do consumo de medicamentos sobre o sistema de saúde brasileiro. Por um lado, a garantia judicial do fornecimento de medicamentos reflete a legitimidade do Poder Judiciário na tutela do direito à saúde (SILVA, 2006). Por outro, a judicialização traz riscos à política de saúde, inerentes à individualização de direitos coletivos, como a possibilidade de distanciamento de critérios técnicos, o que implica no fornecimento, por via judicial, de medicamentos sem eficácia ou segurança comprovadas (BARROSO, 2007; BORGES, 2005).

A propaganda exerce um papel importante quanto ao uso de medicamentos, por influenciar diretamente prescritores e usuários. Com relação ao primeiro grupo, dois problemas interferem na atividade de prescrição. O primeiro é a hegemonia da indústria farmacêutica como fonte de informação sobre medicamentos, com investimentos vultosos em propagandas direcionadas aos médicos. Essa influência, embora quase nunca reconhecida pelos médicos, interfere na prescrição, que passa a ser guiada por outras finalidades, que não a terapêutica (BARROS; JOANY, 2002; BRASIL, 2005). O segundo problema é o crescente financiamento de atividades acadêmicas pela indústria farmacêutica, o que introduz um conflito de interesses nos resultados das investigações e, portanto, na elaboração de guias terapêuticos (BRASIL, 2005). Assim, a demanda por medicamentos de alto custo sem eficácia comprovada, presente nos mandados judiciais, pode estar submetida a uma prescrição conduzida, ora explícita, ora implicitamente, pela propaganda. 
A propaganda de medicamentos está prevista como um dos campos de aplicação dos Critérios Éticos para a Promoção de Medicamentos, documento formulado em 1988 pela Organização Mundial de Saúde, com o objetivo de melhorar a atenção à saúde no que tange ao uso racional de medicamentos (ORGANIZAÇÃO MUNDIAL DA SAÚDE, 1988). No Brasil, a propaganda de medicamentos está regulamentada, especificamente, com relação à vigilância sanitária, no Decreto no 79.094/1977, que submete os medicamentos e outros produtos de interesse para saúde a um sistema de vigilância sanitária. A Agência Nacional de Vigilância Sanitária (Anvisa) publicou, ao longo dos dez anos desde a sua criação, dois marcos regulatórios nesse campo: a Resolução de Diretoria Colegiada (RDC) no 102, de 30 de novembro de 2000, que foi substituída posteriormente pela RDC no 96, de 17 de dezembro de 2008. Elas constituem regulamentos técnicos na área de propaganda, publicidade, promoção e informação de medicamentos.

Observa-se, portanto, que o país possui ampla regulamentação sobre propaganda de medicamentos, que inclui desde dispositivos constitucionais de garantia do direito à saúde até regulamentos técnicos específicos. Entretanto, na prática, problemas como a tendência de crescimento da ocorrência de intoxicaçôes medicamentosas e o alto percentual de propagandas de medicamentos com irregularidades demonstram que as açōes de vigilância sanitária não têm sido capazes de assegurar o controle necessário à proteção da saúde nesse campo de atuação (NASCIMENTO, 2007). Este estudo tem por objetivo analisar o processo de regulamentação da propaganda de medicamentos pela Agência Nacional de Vigilância Sanitária ocorrido em 2008 e identificar os seus resultados quanto ao modelo regulador nessa área de atuação.

\section{Procedimentos e universo de pesquisa}

As mudanças ocorridas em 2008, com a publicação da RDC nº 96/2008, foram estudadas a partir de três fontes de dados. No primeiro, foi realizada uma análise comparativa com a normativa anterior, a RDC no $102 / 2000$. As mudanças mais expressivas foram listadas em duas classes, segundo o conteúdo das normas. Em seguida, as mudanças foram relacionadas a dois documentos técnicos, de escopos diferentes: o primeiro, de alcance mundial, os Critérios Éticos para a Promoção de Medicamentos da Organização Mundial de Saúde; e o segundo, 
de âmbito nacional, a Contribuição ao Debate e Proposições à Consulta Pública 84/2005 da Anvisa, relativa à Propaganda de Medicamentos no Brasil (OFICINA DE TRABALHO SOBRE REGULAÇÃO DA PROPAGANDA DE MEDICAMENTOS NO BRASIL, 2005).

Os Critérios Éticos da Organização Mundial de Saúde nesse tema constituem princípios gerais, a serem adaptados pelos governos, de acordo com as realidades locais. Embora não constituam obrigação jurídica, oferecem indicativos importantes para a tomada de decisão quanto às práticas publicitárias em medicamentos, pois estão guiados pela busca da melhoria da atenção à saúde, por meio do uso racional de medicamentos.

A Oficina de Trabalho sobre Regulação da Propaganda de Medicamentos no Brasil ocorreu em 2005, com a participação de gestores e trabalhadores das instâncias federal e estadual da vigilância sanitária, e de pesquisadores do setor. Em linhas gerais, a oficina teve por objetivo avaliar o modelo regulador vigente de propaganda de medicamentos e propor iniciativas com vistas ao seu aprimoramento. Os resultados foram sistematizados num documento de contribuição à Consulta Pública 84/2005, que teve o apoio de 146 especialistas e de 12 instituições acadêmicas e entidades de representação da sociedade civil. A oficina elaborou propostas de alteração do modelo regulador, a partir da análise da situação de propaganda de medicamentos no país, inclusive por meio da comparação da realidade brasileira à de outros países. O documento representa, portanto, um subsídio para a atividade regulatória, a partir do diagnóstico e da proposição fundamentados a partir de enfoque científico, e das expectativas da sociedade civil quanto à regulação da propaganda de medicamentos.

\section{Resultados}

Dois tipos de mudanças foram identificados a partir da análise comparativa entre a RDC 96/2008 e a normativa anterior, a RDC 102/2000, categorizadas em duas classes, de acordo com o tipo de conteúdo. Os itens não previstos na norma anterior foram considerados como novo conteúdo. Quando se tratou de detalhamento, explicitação ou readequação de regras que já existiam, os itens foram classificados como reformulação de conteúdo. Os resultados segundo as duas classes de conteúdo dessa ordenação legal são apresentados no quadro 1 . 


\section{MUDANÇAS RELATIVAS AO NOVO CONTEÚDO}

1. Proibição de "propaganda ou publicidade enganosa, abusiva e/ou indireta", inclusive por meio de técnicas não declaradamente publicitárias, como as usadas em contexto cênico.

2. Inclusão de medidas mais restritivas ao conteúdo da propaganda ou publicidade, como a proibição do uso de selos de qualidade, de expressóes no imperativo, de imagens de pessoas fazendo uso dos medicamentos e de vinculação com excessos gastronômicos ou etílicos.

3. Inclusão de regras para mensagens dirigidas ao público que contenham informação sobre preços.

4. Ampliação da advertência para medicamentos isentos de prescrição médica. Além da advertência "se persistirem os sintomas, o médico deverá ser consultado", a propaganda deve incluir advertência relacionada à substância ativa, para um grupo de medicamentos especificado na Resolução. Nos demais casos, não contemplados no grupo, deve-se utilizar a advertência "é um medicamento, seu uso pode trazer riscos. Procure o médico e o farmacêutico. Leia a bula." Com relação a esse item, foram definidas regras para a divulgação das advertências.

5. Criação de novos requisitos para amostras grátis, com a proibição de sua distribuição para determinadas classes de medicamentos, e implantação de novas definições para a apresentação das embalagens.

6. Inclusão de requisitos para campanhas sociais e para material informativo de medicamentos manipulados.

7. Estabelecimento de mensagem retificadora, a ser divulgada por empresa infratora após a publicação de decisão condenatória sobre propaganda de medicamentos.

\section{MUDANÇAS DE REFORMULAÇÃO DO CONTEÚDO}

1. A participação de figuras conhecidas do grande público em propagandas foi mantida, desde que não afirme ou insinue que utiliza o remédio, nem recomende o seu uso.

2. Maior definição do limites da propaganda ou publicidade de medicamentos de venda sob prescrição - restrita aos profissionais de saúde habilitados para a sua prescrição ou dispensação -, inclusive quanto à forma de apresentação e origem do conteúdo.

3. A proibição de brindes, benefícios e vantagens se estendeu, além dos profissionais prescritores ou dispensadores, ao público.

Das sete mudanças de novo conteúdo, apenas duas não estão diretamente relacionadas à propaganda dirigida à população, que correspondem aos novos requisitos para amostras grátis, campanhas sociais e material informativo de medicamentos manipulados. Nas demais, há uma evidente atenção quanto à propaganda voltada para o público. Essas medidas aproximam a nova Resolução dos Critérios Éticos para a Promoção de Medicamentos da Organização Mundial de Saúde, em seu critério de publicidade para o público em geral, que estabelece que os anúncios a ele dirigidos "devem contribuir para que a 
população possa tomar decisões racionais sobre a utilização de medicamentos"

(OMS, 1988, p. 8). Na RDC 96/2008, há um extenso detalhamento quanto a expressões e palavras que podem ou não ser utilizadas nos anúncios, o que indica a adoção de critérios restritivos que tornem a propaganda mais próxima de algumas características desejáveis segundo os critérios da Organização Mundial de Saúde: fidedigna, verdadeira, equilibrada e de bom gosto, o que significa que ela não deve conter declarações ou omissões que possam induzir à utilização indiscriminada de medicamentos.

Duas das reformulações no conteúdo da norma estão mais relacionadas aos medicamentos de venda sob prescrição e, portanto, aos profissionais habilitados para a sua prescrição ou dispensação. Forma e conteúdo estabelecidos para esse aspecto da propaganda estão em consonância com os critérios da Organização Mundial de Saúde quanto à publicidade destinada aos profissionais de saúde. O texto e as ilustrações de anúncios dirigidos a profissionais de saúde devem ser inteiramente compatíveis com os dados científicos que constem do registro do medicamento. Isso atende, em alguma medida, às demais características recomendadas pela Organização Mundial de Saúde para a propaganda de medicamentos: exata, informativa, atualizada e suscetível de comprovação (OMS, 1988).

Com relação ao documento com as contribuições resultantes à Consulta Pública no 84/2005, da Oficina de Trabalho sobre Regulação da Propaganda de Medicamentos no Brasil, constata-se que nenhuma das proposições foi atendida. O documento apresenta 19 proposições, com as respectivas justificações, que levam em conta deliberações anteriores à Consulta Pública, como a I Conferência Nacional de Vigilância Sanitária, realizada em 2001. Algumas proposições exigem medidas robustas e de alto impacto regulatório, como a proibição da propaganda de medicamentos, ou o estabelecimento de mecanismos para a sua aprovação prévia pelo Estado. Outras medidas têm dimensão de longo prazo e exigiriam uma profunda reorientação do modelo regulador, como a educação continuada dos profissionais de saúde nesse campo e o envolvimento dos conselhos de saúde das três esferas do governo no controle da propaganda (OFICINA..., 2005).

Além das proposiçóes, o documento apontou cinco problemas graves que deveriam ter sido enfrentados quando da publicação de nova regulamentação para a propaganda de medicamentos: regulação a posteriori; magnitude das irregularidades 
cometidas; multas de valor irrisório; transferência dos valores das multas para o preço dos medicamentos; e uso incorreto de advertência na propaganda (OFICINA..., 2005). Tendo em vista a baixa correlação entre a RDC no 96/2008 e as referidas proposiçōes, é possível afirmar que a normativa não enfrentou essas questôes do modelo regulador da propaganda de medicamentos, limitando-se a efetuar melhorias ou atualização no modelo. Nos últimos anos, a Anvisa tem substituído açôes ligadas ao poder de polícia por ações educativas, no campo da regulação da propaganda. Entretanto, essas açôes, por seu limitado alcance geográfico e baixo impacto sobre o conteúdo das peças publicitárias, parecem ser insuficientes para enfrentar os problemas mencionados (NASCIMENTO, 2007).

A análise de onze mudanças de conteúdo na ordenação legal estabelecida pela RDC no 96/2008 aponta para uma maior proximidade com os Critérios Éticos para a Promoção de Medicamentos da Organização Mundial de Saúde, mas também para um claro distanciamento de proposições para a alteração do modelo regulador atualmente estabelecido. Nesse sentido, é necessário que a estratégia regulatória avance para garantir, entre outros, a promoção de medicamentos a partir de informações fidedignas, equilibradas e passíveis de comprovação, nos termos dos Critérios Éticos da Organização Mundial de Saúde (ORGANIZACIÓN MUNDIAL DE LA SALUD, 1988). Por outro lado, modificações mais profundas no modelo serão necessárias para, além das mudanças de regras, alterar a lógica de veiculação da propaganda de medicamentos, atualmente orientada pelo mercado e descolada de princípios de proteção da saúde.

\section{Considerações finais}

Os resultados aqui apresentados dizem respeito não apenas ao modelo regulador da propaganda de medicamentos, mas à própria ação regulatória da Anvisa, que, por sua vez, expressam os conflitos sobre o papel a ser desempenhado pelo Estado. Eles estão, muitas vezes, relacionados à falta de conexão entre os argumentos teóricos utilizados para justificar a criação de agências reguladoras e o seu desempenho na prática. Assim é que o conceito de regulação social - definido como a proteção do interesse público nas áreas de saúde, segurança, meio ambiente e em questōes nacionais pode colidir com a própria atuação das agências, em seu compromisso com a credibilidade junto ao mercado, que muitas vezes resulta em subserviência 
às suas regras (CRUZ, 2009). A não adoção de medidas mais vigorosas, como a proibição ou a anuência prévia para a propaganda de medicamentos, parece expressar essa dificuldade de conciliar os interesses público e de mercado.

As noções de participação pública e de transparência como requisitos quase que inerentes às agências vêm se deparando com a precariedade de utilização efetiva de mecanismos de controle público de suas decisóes (CRUZ, 2009). Entre esses, a consulta pública, tida como garantia de participação efetiva dos cidadãos, apresenta, na prática, um baixo nível de participação social e baixa representação da sociedade civil nos resultados, o que impõe a sua crítica e revisão. No caso da Consulta Pública no 84/2005, que antecedeu a RDC no 96/2008, a longa discussão da proposta, por um período de três anos, não implicou a assimilação das proposições advindas de técnicos e gestores da área. A permeabilidade à participação da sociedade e a transparência na divulgação dos resultados da análise das contribuiçõos às consultas públicas são desafios a serem enfrentados para o aperfeiçoamento desse mecanismo.

A referida Consulta Pública sofreu muitas críticas por parte de agências de publicidade, veículos de comunicação, indústria farmacêutica e políticos. Os argumentos variaram desde o cerceamento à liberdade de expressão comercial até a suposta incompetência da Anvisa para legislar sobre publicidade. Ao mesmo tempo, diversas entidades participaram do debate, propondo alterações mais substanciais no modelo regulador da propaganda. Essa tensão resultou na paralização do processo de publicação, e, certamente, interferiu nos seus resultados (NASCIMENTO, 2007). A resolução atualiza as regras sobre propaganda de medicamentos isentos de prescrição e sobre vendas sob prescrição. Traz, ainda, requisitos para propaganda ou publicidade em amostras grátis, material informativo de medicamentos manipulados, visitas de propagandistas, eventos científicos e campanhas sociais.

O processo de descentralização das ações de vigilância sanitária para estados e municípios ocorreu de forma mais lenta que no sistema de saúde, por suas dificuldades doutrinárias e de práticas (BRITO, 2007). Isso também se reflete nas atividades de regulação da propaganda, atualmente concentradas na Anvisa, o que repercute no seu alcance populacional e resultados. Um caso ilustrativo dessa dificuldade é a implementação do projeto "Educação para o Consumo Responsável de Medicamentos e de outros Produtos Sujeitos à Vigilância 
Sanitária" - Educanvisa. Uma simulação de seu alcance mostra que seus resultados, caso atingissem seus objetivos em todos os habitantes dos municípios onde foi executado, atingiria cerca de $4 \%$ da população do país (NASCIMENTO, 2007).

Conclui-se que a adoção de medidas que alterem o modelo regulador nesse campo de atuação, o da regulação da propaganda de medicamentos, requer mudanças mais substanciais. Elas pressupōem a revisão do modelo de gestão e da ação regulatória da Anvisa, especialmente quanto ao seu papel de regulação social. Para isso, é necessário que os mecanismos de transparência e de controle social sejam aperfeiçoados e efetivamente utilizados, de forma a qualificar e legitimar a tomada de decisão na regulação em saúde.

\section{Referências}

ANVISA. Resolução de Diretoria Colegiada (RDC) 102/2000. Disponível em http://www. anvisa.gov.br/legis/resol/2000/102_00rdc.htm. Acesso em: 01 maio 2011.

ANVISA. Resolução de Diretoria Colegiada (RDC) 96/2008. Disponível em http://www. anvisa.gov.br/propaganda/rdc/rdc_96_2008_consolidada.pdf. Acesso em: 01 maio 2011.

AQUINO, D.S. Por que o uso racional de medicamentos deve ser uma prioridade? Ciência \& Saúde Coletiva, Rio de Janeiro, v. 13 (Supl.), p. 733-736, 2008.

BARROS, J.A.C. Estratégias mercadológicas da indústria farmacêutica e o consumo de medicamentos. Revista de Saúde Pública, São Paulo, v. 17, p. 377-86, 1983.

BARROS, J.A.C.; JOANY, S. Anúncios de medicamentos em revistas médicas: ajudando a promover a boa prescrição? Ciência \& Saúde Coletiva, Rio de Janeiro, v. 7, p. 891-898, 2002.

BARROSO, R.L. Da falta de efetividade à judicialização excessiva: direito à saúde, fornecimento gratuito de medicamentos e parâmetros para a atuação judicial. Revista Interesse Público, Belo Horizonte, v. 46, p. 1-36, 2007.

BORGES, D.C.L. Uma análise das açôes judiciais para o fornecimento de medicamentos no ámbito do SUS: o caso do Estado do Rio de Janeiro no ano de 2005. 2007. 117 p. Dissertação (Mestrado em Saúde Pública) - Escola Nacional de Saúde Pública Sérgio Arouca, Fundação Oswaldo Cruz, Rio de Janeiro, 2007.

BRASIL. MINISTÉRIO DA SAÚDE. Agência Nacional de Vigilância Sanitária. Estudo comparado: regulamentação da propaganda de medicamentos. Brasília: Ministério da Saúde, 2005.

BRITO, R.L. Análise da politica de descentralização das ações de Vigilância Sanitária no Brasil: do debate sobre o repasse de recursos ao compromisso com a responsabilidade sanitária. Recife. 205 p. Dissertação (Mestrado em Saúde Pública) - Centro de Pesquisas Aggeu Magalhães, Fundação Oswaldo Cruz, Recife, 2007. 
CRUZ, V. Estado e regulação: fundamentos teóricos. In: RAMALHO, P.I.S. (Org.).

Regulação e agências reguladoras: governança e análise de impacto regulatório. Brasília: Anvisa, 2009. p. 53-86.

CUNHA, M.F.C. A racionalidade da mercantilização da doença. 2008.159 p. Dissertação (Mestrado em Saúde Pública) - Faculdade de Saúde Pública, Universidade de São Paulo, São Paulo, 2008.

DANIELS, N.; SABIN, J.E. Our lives in whose hands? In: Setting limits fairly: can we learn to share medical resources. New York: Oxford University Press, 2002. p. 1-12.

MENDONÇA, R.T.; MARINHO, J.L. Discussão sobre intoxicaçôes por medicamentos e agrotóxicos no Brasil de 1999 a 2002. Revista Eletrônica de Farmácia, Goiânia, v. 2, p. 45-63, 2005.

NASCIMENTO, A.C. A persistirem os sintomas o médico deverá ser consultado: isto é regulação? 2003. 124 p. Dissertação (Mestrado em Saúde Pública) - Instituto de Medicina Social, Universidade do Estado do Rio de Janeiro, Rio de Janeiro, 2003.

NASCIMENTO, A.C. Propaganda de Medicamentos. É possível regular? Rio de Janeiro. 289 p. Tese (Doutorado em Saúde Coletiva) - Instituto de Medicina Social, Universidade do Estado do Rio de Janeiro, Rio de Janeiro, 2007.

OFICINA DE TRABALHO SOBRE REGULAÇÃO DA PROPAGANDA DE MEDICAMENTOS NO BRASIL. Contribuição ao debate e proposiçôes à CP 84/2005 da Anvisa. Rio de Janeiro: Escola Nacional de Saúde Pública Sérgio Arouca, Fundação Oswaldo Cruz, 2005. Disponível em <http://www.fiocruz.br/ccs/media/alvaro_nascimento.pdf>. Acesso em: 26 maio 2010.

ORGANIZACIÓN MUNDIAL DE LA SALUD. Criterios éticos para la promoción de medicamentos. Genebra: WHO, 1988.

PFAFFENBACH, G.; CARVALHO, O.M.; BERGSTEN-MENDES, G. Reações adversas a medicamentos como determinantes da admissão hospitalar. Revista da Associação Médica Brasileira, São Paulo, v. 48, p. 237-241, 2002.

ROZENFELD, S. Agravos provocados por medicamentos em hospitais do Estado do Rio de Janeiro, Brasil. Revista de Saúde Pública, São Paulo, v. 41, p. 1-8, 2007.

SEIBERT, S.L. et al. Medicalização x humanização: o cuidado ao parto na história. Revista de Enfermagem, Rio de Janeiro, v. 13, p. 245-251, 2005.

SILVA, F.V.N. Considerações sobre a judicialização do acesso à saúde. Revista da Escola de Direito, Pelotas, v. 68, p. 67-93, 2006.

SYRETT, K. Why 'Ration' Healthcare Resources? In: . Law, legitimacy and the rationing of health care. Cambridge: Cambridge University Press, 2007. p. 15-44.

TESSER, C.D. Medicalização social (I): o excessivo sucesso do epistemicídio moderno na saúde. Interface - Comunicação, Saúde, Educação, São Paulo, v. 9, p. 61-76, 2006. 
New rules and old challenges in the regulation of drug advertising

This study aimed to analyze the process of regulation of drug advertising by ANVISA (National Health Surveillance Agency) in 2008 and to identify its results on the regulatory model in this area. We analyzed the changes in 2008 with the publication of RDC No. $96 / 2008$, based on three data sources. The first was a comparative analysis with the previous rules, the RDC No. 102/2000. Then, the changes were related to two technical papers in different scopes: the Ethical Criteria for Drug Promotion of the World Health Organization, with global reach; and the Contribution to the Debate and Proposals for Public Consultation 84/2005, nationwide, issued by ANVISA, on drug advertising in Brazil. The analysis of 11 content changes in the legal order established by RDC No. 96/2008 points to a closer relationship with the Ethical Criteria for Promotion of Medicines of the World Health Organization, but a clear separation of proposals for changing the regulatory model currently established. It was concluded that the adoption of measures amending the regulatory model in this field, the regulation of drug advertising, requires more substantial changes in the management model and the regulatory action of ANVISA, especially regarding the role of social regulation.

> Key words: drug advertizing; health surveillance; regulation. 